\title{
MINIMAL REGULAR SPACES
}

\author{
MANUEL P. BERRI AND R. H. SORGENFREY
}

1. Introduction. If $\odot$ is a property of topologies, a space $(X, J)$ is minimal $\rho$ if $J$ has property $\rho$, but no topology on $X$ which is strictly weaker $(=$ smaller $)$ than $J$ has $P$. Such spaces have been investigated for the case $\beta=$ Hausdorff $[2 ; 5]$, a well-known result being that while every compact space is minimal Hausdorff, the converse is not true. We consider here the case $P=$ regular $;^{1}$ other properties are discussed by one of the authors in a paper to appear.

Filter-bases on spaces will be used extensively (for definitions not given here, see [1]). A filter-base is open (closed) if its elements are open (closed) sets. A filter-base will be called regular if it is open and is equivalent to a closed filter-base. The name is suggested by the fact that the filter-base of open neighborhoods of a point of a regular space is regular since it is equivalent to the filter-base of closed neighborhoods of that point.

2. Characterizations of minimal regular spaces. We will be concerned with spaces satisfying one or both of the following conditions:

$(\alpha)$ Every regular filter-base which has a unique adherent point is convergent.

$(\beta)$ Every regular filter-base has an adherent point.

Theorem 1. A regular space which satisfies $(\alpha)$ also satisfies $(\beta)$.

Proof. Suppose $B$ is a regular filter-base on the regular space $(X, J)$ and that $B$ has no adherent point. Let $\mathcal{C}$ be a closed filter-base equivalent to $B$. Fix $p \in X$ and let $\mathcal{u}$ and $v$ be the filter-bases of open and closed neighborhoods of $p$, respectively. Since $\mathfrak{J}$ is regular, $\mathfrak{u}$ and $\vartheta$ are equivalent. Then $R=\{B \cup U: B \in B, U \in \mathcal{u}\}$ is an open filterbase equivalent to the closed filter-base $\{C \cup V: C \in \mathbb{e}, V \in \mathcal{U}\}$ and is therefore reguldr. It is clear that $p$ is the unique adherent point of $R$ and that $R$ does not converge to $p$. This denial of the hypothesis establishes the theorem.

TheOREM 2. In order that a regular space be minimal regular, it is necessary and sufficient that it satisfy $(\alpha)$.

Proof. Suppose $(X, J)$ is regular and that $B$ is a regular filter-base having the unique adherent point $p$ to which it does not converge.

Presented to the Society, October 30, 1961; received by the editors March 12,1962.

${ }^{1}$ As used in this paper, the condition of regularity includes $T_{1}$ separation, i.e., singletons are closed. 
For each $x \in X$, let $\mathfrak{u}(x)$ be the filter-base of J-open neighborhoods of $x$ and define $\mathcal{u}^{\prime}(x)=\mathfrak{u}(x)$ if $x \neq p$ and $\mathfrak{u}^{\prime}(p)=\{U \cup B: U \in \mathcal{u}(x)$, $B \in B\}$. There is a topology $J^{\prime}$ on $X$ such that $\mathfrak{u}^{\prime}(x)$ is an open base at $x$ for each $x \in X$. It is clear that $J^{\prime}$ is strictly weaker than $J$ (there is a $U \in \mathcal{u}(p)$ which contains no set of $\mathcal{u}^{\prime}(p)$ since $B$ does not converge to $p$ ). Moreover, $J^{\prime}$ is certainly regular at each $x \neq p$, while regularity at $p$ follows readily from the fact that $B$ is equivalent to a closed filter-base. Hence $J$ is not minimal regular.

To establish the sufficiency of the condition, let $(X, \Im)$ be a regular space satisfying $(\alpha)$ and let $J^{\prime}$ be a regular topology on $X$ which is weaker than $\mathfrak{J}$. For arbitrary $x \in X$ let $\mathfrak{u}(x)$ and $\mathfrak{u}^{\prime}(x)$ be the open neighborhood systems of $x$ in the $J$ and $J^{\prime}$ topologies, respectively. The filter-base $\mathcal{U}^{\prime}(x)$ is $J^{\prime}$-regular and has $x$ as its only adherent point. Since $J^{\prime}$ is weaker than $J, \mathcal{U}^{\prime}(x)$ is regular and has unique adherent point $x$ in $(X, \mathfrak{J})$. By $(\alpha) \mathcal{U}^{\prime}(x)$ converges to $x$ in $(X, \mathfrak{J})$. Hence $\mathcal{U}(x)$ must be weaker than $\mathcal{U}^{\prime}(x)$, and, since the reverse is true, it follows that $J$ and $J^{\prime}$ are identical and that $J$ is minimal regular.

REMARK. The two previous results show that condition $(\beta)$ is necessary in order that a regular space be minimal regular. Whether it is sufficient is an open question. Theorem 3 below, however, throws some light on the problem.

Lemma. If the subspace $X$ of the regular space $Y$ satisfies $(\beta)$, then $X$ is closed in $Y$.

Proof. Suppose $p \in \bar{X}-X$. Let $\mathcal{u}$ and $v$ be, respectively, the open and closed neighborhood systems of $p$ in $Y$. Then the filter-base $B=\{X \cap U: U \in \mathcal{u}\}$ is open (relative to $X$ ), is equivalent to the closed (relative to $X$ ) filter-base $\{X \cap V=V \in \mathcal{V}\}$, and is therefore regular on $X$. As a filter-base on $Y, \otimes$ is stronger than $\mathcal{u}$ and hence has no adherent point other than $p$ in $Y$. It follows that $\beta$ has no adherent point at all in $X$, a contradiction.

THeORem 3. Any completely regular space satisfying $(\beta)$ is compact and therefore minimal regular.

Proof. Let $X$ be completely regular and satisfy $(\beta)$ and let $Y$ be its Stone-Cech compactification. The above lemma yields the desired result.

THEOREM 4. Any minimal regular subspace of a regular space is closed.

Proof. This is an immediate consequence of the lemma since the subspace must satisfy $(\beta)$. 
REMARK. It is easy to see that a subspace of a minimal regular space which is both open and closed is itself minimal regular. The example of the next section shows that a closed subspace of a minimal regular space need not be minimal regular.

3. A minimal regular noncompact space. The example given here is a slight modification of an unpublished one due to Richard Arens of a regular space which is not completely regular. His example has also been used by Hewitt [3] in constructing a regular space on which every continuous real-valued function is constant.

Description of the space $(Z, J)$. Let $J$ be the set of all integers, $\omega^{\prime}$ the ordinals $\leqq \omega$, and $\Omega^{\prime}$ the ordinals $\leqq \Omega$ (the first uncountable one). Equip each of these sets with the order topology and consider the space $J \times \omega^{\prime} \times \Omega^{\prime}-\{(n, \omega, \Omega): n \in J\}$, the relative product topology being used. To obtain the space $Y$, make the following identifications and use the quotient topology $J^{*}$ : for even $n$, identify $(n, \omega, y)$ and $(n+1, \omega, y)$; for odd $n$, identify $(n, x, \Omega)$ and $(n+1, x, \Omega)$. We will continue to use the symbols $(n, x, y)$ for the points of $Y$, thus $(n, \omega, y)$ $=(n+1, \omega, y)$ for even $n$. For $n \in J$, let $Q_{n}=\{(n, x, y): x<\omega, y<\Omega\}$ and $Z_{n}=\bar{Q}_{n}=\{(n, x, y):(x, y) \neq(\omega, \Omega)\}$. Let $p$ and $q$ be points not in $Y$ and topologize $Z=\{p\} \cup\{q\} \cup Y$ by letting an open base at $p$ be all sets of the form

$$
V_{n}(p)=\cup\left\{Z_{i}: i>n\right\} \cup Q_{n} \cup\{p\}, \quad n=1,2, \cdots,
$$

and an open base at $q$ be all sets of the form

$$
V_{n}(q)=\cup\left\{Z_{-i}: i>n\right\} \cup Q_{-n} \cup\{q\}, \quad n=1,2, \cdots,
$$

which open bases at points of $Y$ are those they had in $3^{*}$. Let the resulting topology on $Z$ be $\mathfrak{J}$.

Properties of the space $(Z, \Im) .1 .(Z, J)$ is regular.

Proof. It is easy to see that singletons are closed, and regularity is clear except possibly at $p$ and $q$. Regularity at $p$, say, follows from $\left[V_{n+1}(p)\right]-\subset V_{n}(p)$.

We will say that a set $S \subset Z$ gets into the $n$-corner if whenever $x_{0}<\omega, y_{0}<\Omega$, there is a point $(n, x, y) \in S$ for some $x>x_{0}$ and $y>y_{0}$.

2 . If the open set $U$ gets into the $n$-corner, then there is an infinite sequence $\left\{x_{i}\right\}$ of distinct finite ordinals such that $\left(n, x_{i}, \Omega\right) \in \bar{U}$.

Proof. If not, there is an $x_{0}<\omega$ such that if $x_{0}<x<\omega,(n, x, \Omega) \in \bar{U}$ and hence there is a $y_{x}<\Omega$ such that $(n, x, y) \notin U$ for $y_{x}<y$. Since $\left\{y_{x}: x_{0}<x<\omega\right\}$ is countable, its least upper bound, $y_{0}$, is less than $\Omega$. Therefore if $x_{0}<x<\omega$ and $y_{0}<y$, then $(n, x, y) \notin U$. Since $U$ gets into the $n$-corner, it must then be that $(n, \omega, y) \in U$ for some $y>y_{0}$. But 
since $U$ is open, there is an $x, x_{0}<x<\omega$, such that $(n, x, y) \in U$. This contradiction establishes the property.

3. Let $U, V$, and $W$ be open sets such that $U \subset \bar{U} \subset V \subset \bar{V} \subset W$. Then if $U$ gets into the $n$-corner, $W$ gets into the $(n-1)$ - and $(n+1)$ corners.

PROOF FOR $n$ ODD. (The proof for the case $n$ even is similar.) Take $x_{0}<\omega, y_{0}<\Omega$. By property 2 , there are infinitely many distinct $x_{i}$ such that $x_{0}<x_{i}<\omega$ and $\left(n, x_{i}, \Omega\right) \in \bar{U}$. Since $\left(n+1, x_{i}, \Omega\right)=\left(n, x_{i}, \Omega\right)$ $\in \bar{U} \subset W, W$ gets into the $(n+1)$-corner. Since $\left(n, x_{i}, \Omega\right) \in V$, there exists, for each $i$, a $y_{i}<\Omega$ such that if $y>y_{i}$, then $\left(n, x_{i}, y\right) \in V$. Let $y^{\prime}$ be the least upper bound of the set $\left\{y_{0}, y_{1}, y_{2}, \cdots\right\}$. Then for any $y, y^{\prime}<y<\Omega,\left(n, x_{i}, y\right) \in V$ for all $x_{i}$; hence $(n, \omega, y)=(n-1, \omega, y)$ $\in \bar{V} \subset W$, and $W$ gets into the $(n-1)$-corner.

4. If $B$ is a regular filter-base and, for some $n$, each set of $B$ gets into the $n$-corner, then $p$ and $q$ are adherent points of $B$.

Proof. Let $N$ be a neighborhood of $p$ and $B \in ß$. There is an integer $k$ such that $Q_{k} \subset V_{k}(p) \subset N$; let $h=k-n$. Since $B$ is regular, there are $2 h+1$ sets $U_{i} \in B$ such that

$$
U_{1} \subset \bar{U}_{1} \subset U_{2} \subset \bar{U}_{2} \subset \cdots \subset U_{2 h+1}=B .
$$

Since $U_{1}$ gets into the $n$-corner, $h$ applications of property 3 shows that $B=U_{2 h+1}$ gets into the $n+h=k$-corner; i.e., $B \cap Q_{k} \neq 0$, whence $B \cap N \neq 0$, and $p$ is an adherent point of $B$. The case for $q$ is similar.

5. $(Z, J)$ is not completely regular and hence not compact.

Proof. Let $f$ be a bounded, real-valued continuous function on $Z$. For some fixed $n$ and each $y<\Omega$, let $g(y)=f(n, \omega, y)$. Then $g$ is continuous, and it is well-known (e.g., [4, p. 167, ex. Q]) that there is a $y_{0}<\Omega$ and a constant $c$ such that $g(y)=c$ for $y>y_{0}$. It follows that each set of the regular filter-base $\{\{p \in Z:|f(p)-c|<\epsilon\}: \epsilon>0\}$ gets into the $n$-corner. Since, by property $4, p$ and $q$ are adherent points of this filter-base, it is clear that $f(p)=f(q)=c$ and $(Z, J)$ is not completely regular.

In the proof of the following property we repeatedly use the elementary fact that if $B$ is a regular filter-base and $C \in B$, then $\mathfrak{C}=\{C \cap B: B \in B\}$ is a regular filter-base equivalent to $B$. We will call $\mathcal{C}$ the $C$-section of $B$.

6. $(Z, J)$ is minimal regular.

Proof. Let $B$ be a regular filter-base with unique adherent point $r$. We will show that $B$ converges to $r$; the property will then follow from Theorem 2 .

Case 1. $r \neq p, q$. Then some set $C \in ß$ meets only a finite number of $Z_{n}$ 's. Let $\mathcal{C}$ be the $C$-section of $B$; then there is an integer $k$ such that 
each set of $\mathcal{C}$ is a subset of $K=\bigcup\left\{z_{n}:|n| \leqq k\right\}$. It follows from property 4 that for each $n,|n| \leqq k$, there is a set $D_{n} \in \mathcal{C}$ which does not get into the $n$-corner. Let $D$ be a set of $\mathcal{e}$ lying in $\cap\left\{D_{n}:|n| \leqq k\right\}$; then ordinals $x_{0}<\omega, y_{0}<\Omega$ exist such that $D$ does not meet the open set $W=\left\{(n, x, y): x>x_{0}, y>y_{0}\right\}$. Hence $D$, the $D$-section of $\mathcal{e}$, is a filter-base equivalent to $B$, and each of its sets lies in the compact subspace $K-W$ of $Z$. It is clear that $D$, and hence $B$, must converge to their unique adherent point $r$.

Case 2. $r=p$. (The proof for the case $r=q$ is similar.) If $B$ does not converge to $p$, there is a neighborhood $V_{k}(p)$ which contains no set of $B$. Since $q$ is not an adherent point of $B$, there is an integer $h$ and a set $C$ of $B$ such that $C \cap Z_{n}=\varnothing$ for $n<h$. It follows from property 4 that for each $n, h \leqq n \leqq k$, there is a set $D_{n}$ in the $C$-section $\mathcal{C}$ of $B$ which does not get into the $n$-corner. Let $D$ be a set of $\mathcal{C}$ lying in $\cap\left\{D_{n}: h \leqq n \leqq k\right\} ;$ then ordinals $x_{0}<\omega$ and $y_{0}<\Omega$ exist such that $D$ does not meet the set $W=\left\{(n, x, y): h \leqq n \leqq k, x>x_{0}, y>y_{0}\right\}$. The $D$-section $D$ of $\mathcal{C}$ is a filter-base equivalent to $B$ and each of its sets meets the compact set $F=\bigcup\left\{Z_{n}: h \leqq n \leqq k\right\}-W$. Hence $\mathcal{E}=\{F \cap E: E \in D\}$ is a filter-base stronger than $B$ and each of its sets is contained in $F$. Since $F$ is compact, $\mathcal{E}$, and hence $B$, must have an adherent point $z \in F$. Since $z \neq p$, a contradiction results.

7. $(Z, J)$ has a closed subspace which is not minimal regular.

Proof. Let $S=\{(1, x, \Omega): x<\omega\}$. It is clear that $S$ is a closed subset of $Z$. But, with the relative topology, $S$ is an infinite discrete space, which is certainly not minimal regular.

\section{REFERENCES}

1. N. Bourbaki, Topologie gênérale, Actualités Sci. Ind., Hermann, Paris, 1951, $858-1142$.

2. - Espaces minimaux et espaces completement séparés, C. R. Acad. Sci. Paris 205 (1941), 215-218.

3. E. Hewitt, On two problems of Urysohn, Ann. of Math. (2) 47 (1946), 503-509.

4. J. L. Kelley, General topology, Van Nostrand, New York, 1955.

5. A. Ramanathan, Maximal Hausdorff spaces, Proc. Indian Acad. Sci., Sect. A 26 (1947), 31-42.

University of California, Los Angeles 